\title{
Evaluation of the Antifungal Activity of Sulfur and Chitosan Nanocomposites with Active Ingredients of Ruta graveolens, Thymus vulgaris and Eucalyptus melliodora on the Growth of Botrytis fabae and Fusarium oxysporum
}

Aguirre V Yela*, Villarreal J Jimenez, Delgado V Rodriguez and Gavilanez P Quishpe

Department of Life Sciences and Agriculture, Universidad de las Fuerzas Armadas - ESPE, CENCINAT, Sangolqui , Ecuador

"Corresponding author: Aguirre V Yela, Department of Life Sciences and Agriculture, Universidad de las Fuerzas Armadas - ESPE, CENCINAT, Sangolqui, Ecuador, Tel: +39 +81566 5804; E-mail: vaaguirre@espe.edu.ec

Received date: March 28, 2016; Accepted date: May 20, 2016; Published date: May 25, 2016

Copyright: (c) 2016 Yela AV, et al. This is an open-access article distributed under the terms of the Creative Commons Attribution License, which permits unrestricted use, distribution and reproduction in any medium, provided the original author and source are credited.

\begin{abstract}
The purpose of this research was to determine the inhibitor effects of nanocomposites over two pathogenic fungi: Botrytis fabae Sard and Fusarium oxysporum isolated from bean crops. These nanocomposites were prepared with elemental sulfur nanoparticles coated with essential oils (four levels, three oils) and further stabilized with chitosan. The research was conducted in the microbiology laboratory of CENCINAT. We evaluated 12 nanocomposites at room temperature and PDA plates at $\mathrm{pH} 4$ for 10 days and compared to controls with no nanocomposites additions. Data for statistical analysis were gathered starting from the seventh growth day because of the fungi efficient growth without treatment is between the fourth and fifth day, respectively. Statistical experimental analysis were carried out through a complete randomized block design using each Petri dish, with spots with 4 nacomposites in each spot. A total of 12 final different nanocomposites were used for all assayed plates giving five repetitions, four replicates and two fungi to carry out ANOVA analysis. Significant differences measures on fungal growth in each treatment, were compared according to Docimo Dunca's multiple range for $p<0.05$ using the statistical package InfoStat Professional. The independent analyzed variables were Fusarium oxysporum, Botrytis fabae Sard and different essential oils produced by Ruta graveolens, Eucalyptus melliodora, Thymus vulgaris and concentrations of each oil $(20 \%, 40 \%, 60 \% .80 \%)$. The results indicate that the nanocomposites application over the fungi in vitro could be effective according to the fungi species. $F$. oxysporum is the most inhibited by applying the nanocomposite with common rue or thyme oil $40 \%$ (optimum concentration), while $60 \%$ and $80 \%$ did not show any statistical difference. However, B. fabae inhibition was shown at these 60 and $80 \%$ concentrations. The nanocomposites with $20 \%$ eucalyptus displayed smaller inhibition halos against both fungi.
\end{abstract}

Keywords: Fusarium oxysporum; Botrytis fabae Sard; Nanocomposites; Eucalyptus; Thyme; Common rue; Bean

\section{Introduction}

Beans, Vicia faba L., are the oldest known legume since production dates back to prehistoric times. It is native to the Mediterranean region especially Iran and Italy. The largest area devoted to beans growing in Ecuador is the Chimborazo province. In our study we have focused on Vicia faba L. bean, cultivation in the Community of Galte Laime Chimborazo province. Crop losses in this region are due to diseases caused by pathogenic fungi such as Fusarium oxysporum and Botrytis fabae Sard which generate serious damage to agricultural level in the pre and post-harvest bean stages affecting economy of these populations.

F. oxysporum is a fungus capable of secreting enzymes and small proteins during colonization of plant xylem vessels that promotes plant invasion. This fungus survives as sclerotia, when favorable weather conditions arise, conidia forms, germinates and spread by rain and wind to infect new plants [1]. B. fafae also is able to penetrate and invade the host tissue by the action of enzymes that degrade the cell wall. Pectinases, cellulases and hemicellulases are the main enzymes involved in pathogen infection, among these we can find: pectinmethylesterases, endopolygalacturonase, exopolygalacturonase, laccases, xilanosa and arabinose, among others [2].

Synthetic pesticides are widely used to control pests and plant diseases, but its indiscriminate application has caused various problems, such as accumulation of toxic residues in treated products, environmental pollution and resistance developed by pests to these pesticides. This research proposes the development of a technology that includes (i) Isolating the fungus Botrytis fabae Sard and Fusarium oxysporum from leaf samples of $V$. faba L crops in the Galte Laime community - Chimborazo - Ecuador (ii) extracting essential oils by steam distillation method of rue (Ruta graveolens), eucalyptus (Eucalyptus melliodora), thyme (Thymus vulgaris) (iii) synthesize nanoparticles of elemental sulfur and make nanocomposites, and (iiii) evaluate in vitro the antifungal activity of the nanocomposites on growth of fungi Botrytis fabae Sard and Fusarium oxysporum.

\section{Methodology}

\section{Culture medium preparation}

Potato Dextrose Agar (modified PDA) was prepared as follow: 23.4 grams of PDA are added to $1 \mathrm{~L}$ BOECO amber bottle, diluted to 1000 $\mathrm{ml}$ with distilled water and stirred up to complete dissolution and 
Citation: Yela AV, Jimenez VJ, Rodriguez DV, Quishpe GP (2016) Evaluation of the Antifungal Activity of Sulfur and Chitosan Nanocomposites with Active Ingredients of Ruta graveolens, Thymus vulgaris and Eucalyptus melliodora on the Growth of Botrytis fabae and Fusarium oxysporum. Biol Med (Aligarh) 8: 291. doi:10.4172/0974-8369.1000291

Page 2 of 4

further sterilized for 30 minutes. The sterilized media was cooled to about $50^{\circ} \mathrm{C}$ within a laminar flow chamber and Levofloxacin, $500 \mathrm{mg}$ was added. The media was then dispensed in $20 \mathrm{~mL}$ Petri dishes to allow medium solidification.

Isolation of fungal pathogens: Plant samples with symptoms of chocolate spot and root rot were gathered, taking into account the type of injury, size and color. To isolate fungi from infected plant the gathered plant infected material was washed with running water, dried with towel paper, then the edges of the lesion were cut and treated with ethanol 70\% for two minutes Samples were then inoculated on PDA medium amended agar and incubated for eight days at room temperature; Microscopic fungi were identified. Each week Botrytis fabae and Fusarium oxysporum plates were replicated to maintain a microbial bank used during the investigation.

\section{Extraction of essential oils}

Samples of common rue, eucalyptus and thyme are collected and extraction of essential oils is done using the steam method; analyzing density, refractive index, $\mathrm{pH}$ etc.

\section{Chitosan preparation}

$0.5 \mathrm{~g}$ of chitosan were dissolved in $100 \mathrm{~mL}$ of $3 \%$ acetic acid, allowed to stir at $1000 \mathrm{rpm}$. During three days and refrigerate for later use.

\section{Sulfur nanoparticle preparation}

In a $0.01 \mathrm{M} \mathrm{Na} 2 \mathrm{~S} 2 \mathrm{O} 3.5 \mathrm{H} 2 \mathrm{O}$ solution is added $\mathrm{HCl}$ in a $2: 1$; the redox reaction occurs at $50 \mathrm{~min}$. and the nanoparticles are ready for use, its diameter is about $7 \mathrm{~nm}$.

\section{Preparation of nanocomposites}

Nanocomposites are obtained from the combination of nanoparticles of sulfur, essential oils (thyme, eucalyptus and common rue) at different concentrations $(20 \%, 40 \%, 60 \%, 80 \%)$ and chitosan. In a relationship 30:7:5, respectively, its diameter is about $300 \mathrm{~nm}$.

\section{Evaluation of the antifungal activity of nanocomposites}

A small amount of pure culture of the fungus is taken with a sterile swab and seeded in a PDA amended medium, extending across the surface of the Petri dish. Four $6 \mathrm{~mm}$ discs are deposited on the surface of the inoculated culture medium is correct distance from each other, is placed over each $10 \mu \mathrm{l}$ of the 12 nanocomposites formed and allowed to incubate for 5 days. 5 repetitions for each nanocomposite are performed.

\section{Results}

F. oxysporum was identified but with a very variable morphology, the production of abundant aerial mycelium is observed cottony coloration from white to pink peach, with few microconidia and more intense violet dye into the agar surface as depicted in Figures $1 \mathrm{~A}$ and 2B.

Botrytis fabae Sard in PDA medium grows and develops into white cottony structures and turn gray as it matures three to six days grows nine centimeters as shown in Figures $1 \mathrm{~B}$ and $2 \mathrm{~A}$.
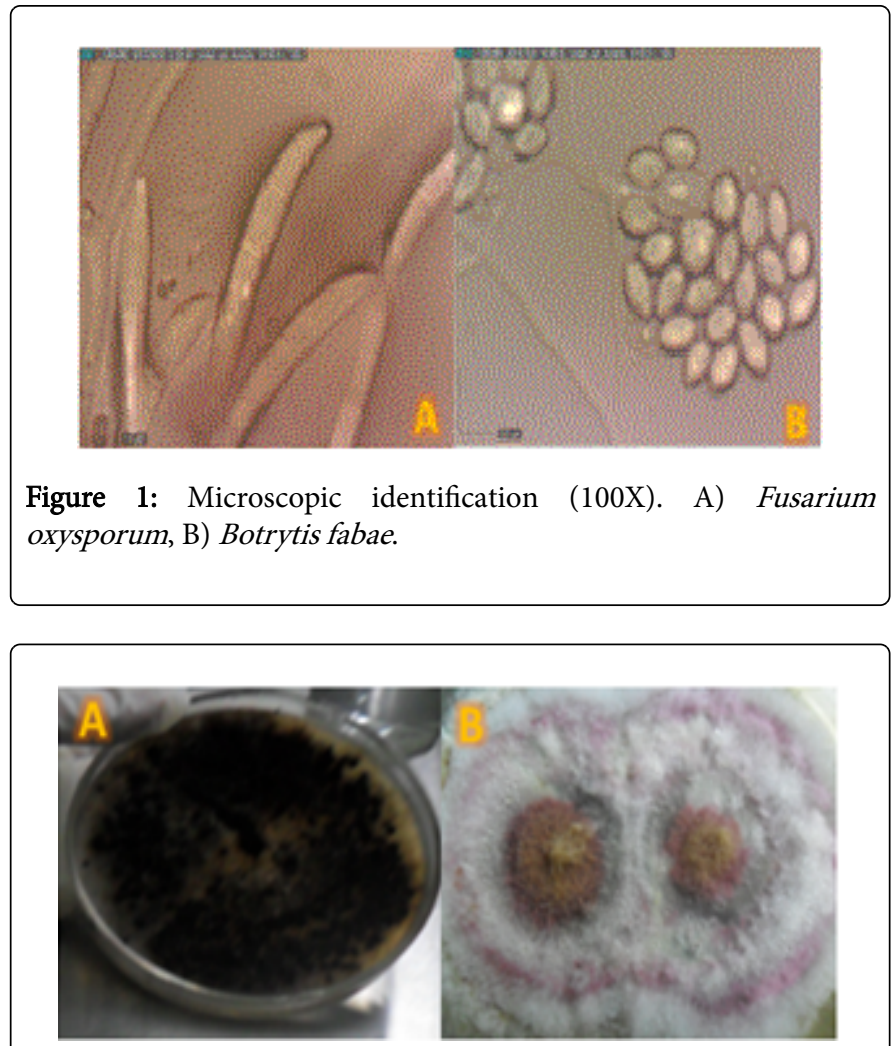

Figure 2: Fungal growth and morphology at 25 days. A) Botrytis fabae, B) Fusarium oxysporum.

The next step was the preparation of sulfur nanoparticles, the reaction time between the sodium thiosulfate pentahydrate and hydrochloric acid was about 50 minutes. The solution takes on a reddish color and the nanoparticles are ready to be used, as shown in Figure 3 after transmission electronic microscopy (TEM).

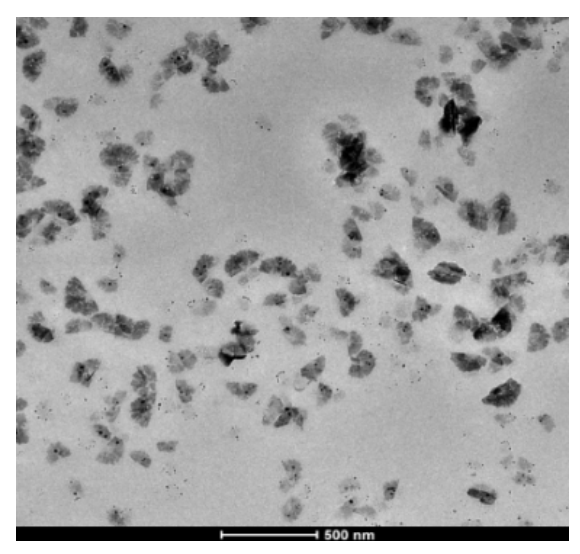

Figure 3: Sulphur nanoparticles observed by TEM. 
Citation: Yela AV, Jimenez VJ, Rodriguez DV, Quishpe GP (2016) Evaluation of the Antifungal Activity of Sulfur and Chitosan Nanocomposites with Active Ingredients of Ruta graveolens, Thymus vulgaris and Eucalyptus melliodora on the Growth of Botrytis fabae and Fusarium oxysporum. Biol Med (Aligarh) 8: 291. doi:10.4172/0974-8369.1000291

Figure 4 shows the synthesized nanocomposites at different concentrations of essential oils to prevent the growth of Fusarium oxysporum and Botrytis fabae.

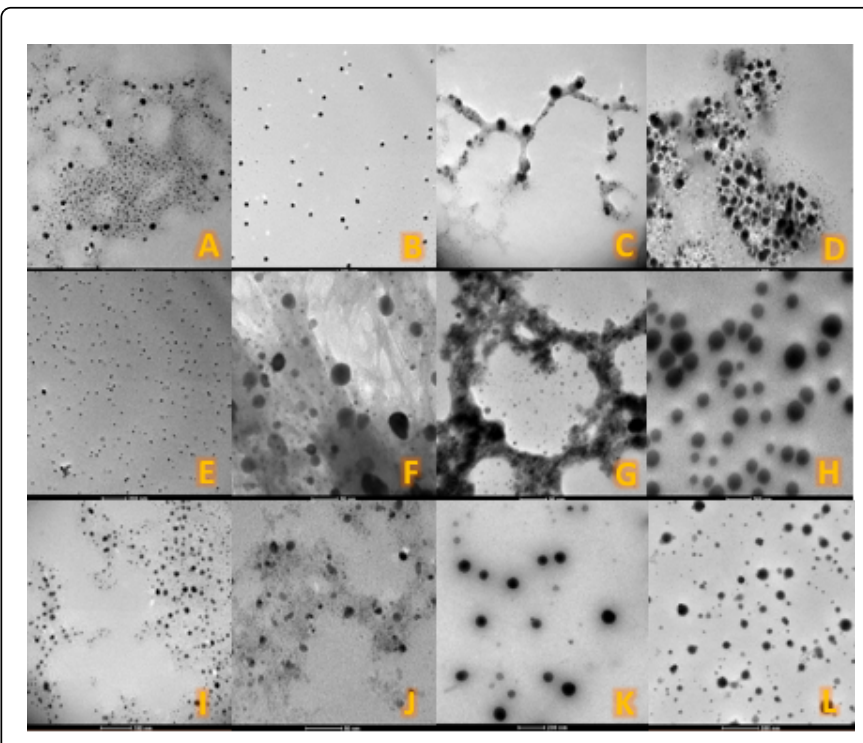

Figure 4: Nanocomposites with essential oils at different concentrations observed in TEM. Common rue essential oil, A) $20 \%$ B) $40 \%$ C) $60 \%$ D) $80 \%$. Thyme essential oil, E) $20 \%$ F) $40 \%$ G) $60 \%$ H) $80 \%$, Eucalyptus essential oil I) $20 \%$ J) $60 \%$ K) $60 \%$ L) $80 \%$.

Figure 5 shows the antifungal effect of $40 \%$ thyme essential oil over Fusarium oxysporum. The inhibition halos can be observed in the Figure.

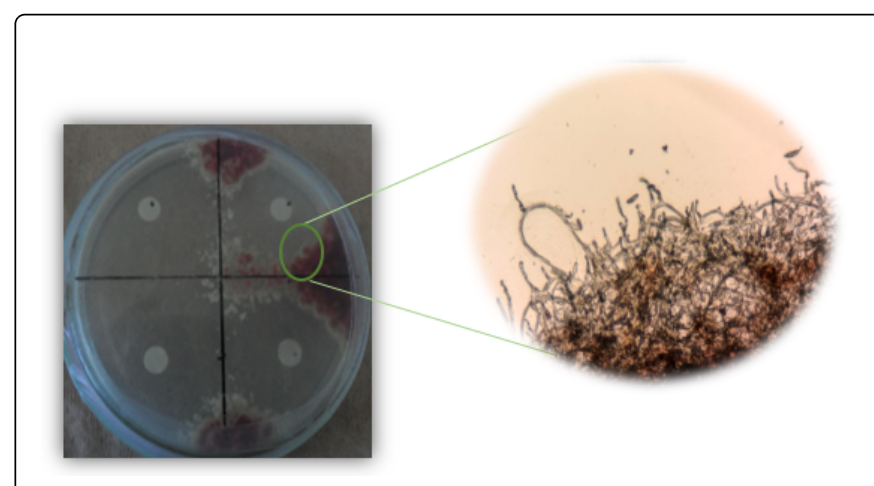

Figure 5: Antifungal effect of $40 \%$ thyme essential oil over Fusarium oxysporum.

Growth inhibition of Botrytis fabae using nanocomposites with $40 \%$ thyme essential oil is shown in Figure 6. This Figure clearly demonstrate the antifungal effect of these nanocomposites.

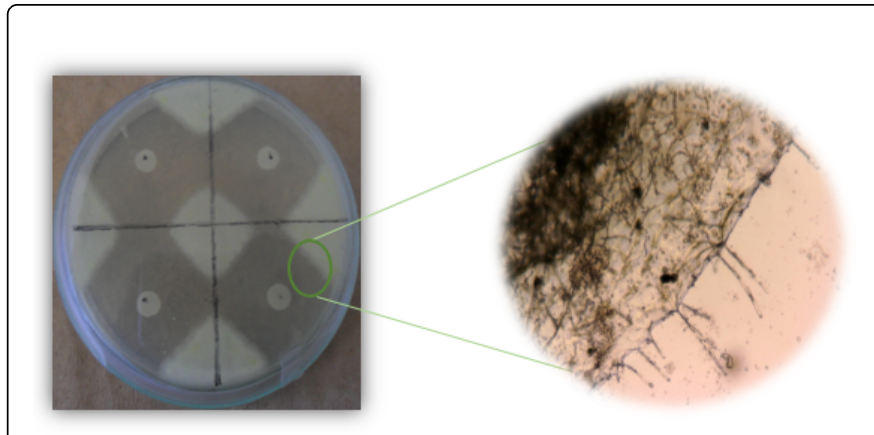

Figure 6: Antifungal effect of nanocomposites with $40 \%$ thyme essential oil over Botrytis fabae.

\section{Conclusions}

- With the steam method, the highest performance to build nanocomposites was obtained on the thyme sample corresponding to $0.81 \%$.

- Nanocomposites structured with thyme oil showed greater sphericity than those displayed by common rue and eucalypti which have rough and irregular structures.

- The inhibition produced by the nanocomposite with thyme essential oil at $40 \%$ is the most effective in removing Fusarium oxysporum and Botrytis fabae.

- The area/surface ratio allows to demonstrate that irregular structures are those that interact with the cell membrane of fungi and are effective at inhibiting their growth.

\section{Discussion}

Plants fungal pathogens have strategies to recognize the appropriate host, penetrate and invade plant tissue, overcome plant defenses and optimize its growth within the host [3]. To perform these processes, usually, fungus perceive chemical and physical signals and host metabolic respond so, morphogenetic changes are required for pathogenic development. Such changes directly include hyphae growth, plant surface adhering, differentiation of specialized infection structures and secretion of lytic enzymes and phytotoxins [4].

Nanocomposites and essential oils have become now-a-days very popular since they can be used as an alternative to be applied to different crops in order to control different plant deseases like bean chocolate spot and root rot. These strategies environmentally friendly, should solve in a cost effective manner farmers problems with the attack of these fungi, reducing production costs and lowering the use of pesticides. Chitosan, used for essential oils nanoencapsulation, has not only stabilizing or antioxidant properties also, by a process of permeabilized energy, penetrates the plasma membrane of different fungal cells (hyphae, spores and germ tubes). This fact suggest interaction with external membrane components causing conformational changes involving pore formation and subsequently entering essential oil and sulfur nanoparticles [5].

However, as shown in this report, further studies must point to study the metabolic pathways of essential oils and using in vitro cultures for improving the performance of active ingredients for use as a structural part of the nanocomposites in order to increase its 
Citation: Yela AV, Jimenez VJ, Rodriguez DV, Quishpe GP (2016) Evaluation of the Antifungal Activity of Sulfur and Chitosan Nanocomposites with Active Ingredients of Ruta graveolens, Thymus vulgaris and Eucalyptus melliodora on the Growth of Botrytis fabae and Fusarium oxysporum. Biol Med (Aligarh) 8: 291. doi:10.4172/0974-8369.1000291

Page 4 of 4

antifungal effect. Also, it should be established the stability of nanocomposites at different temperatures and relative humidities, raising the $\mathrm{pH}$ to prevent soil acidification when applied into the field.

\section{Acknowledgement}

Authors thanks the valuable help of Dr. Luis E. Trujillo, Head of Industrial Biotechnology and Bioproducts research group of the Universidad de las Fuerzas Armadas, ESPE by the critical reading of this short report.

\section{References}

1. Houterman PM, Speijer D, Dekker HL, DE Koster CG, Cornelissen BJ, et al. (2007) The mixed xylem sap proteome of Fusarium oxysporuminfected tomato plants. Mol Plant Pathol 8: 215-221.
2. Kars I, Van Kan A (2007) Extracellular enzymes and metabolites involved in pathogenesis of Botrytis. En Botrytis biology, pathology and control, Springer.

3. Di Pietro A, García-MacEira F, Méglecz E, Roncero MI (2001) A MAP kinase of the vascular wilt fungus Fusarium oxysporum is essential for root penetration and pathogenesis. Mol Microbiol 39: 1140-1152.

4. Bosland PW (2010) Fusarium oxysporum a pathogen of many plant species. En Advances in plant pathology pp: 281-289.

5. Palma-Guerrero J, Jansson HB, Salinas J, Lopez-Llorca LV (2008) Effect of chitosan on hyphal growth and spore germination of plant pathogenic and biocontrol fungi. J Appl Microbiol 104: 541-553.

This article was originally published in a special issue, entitled: "Recent 